\title{
Management of multiple meningiomas
}

\author{
Georgios Tsermoulas, MUDr, MSc, FRCS(SN), ${ }^{1}$ Mazda K. Turel, MCh, ${ }^{1}$ Jared T. Wilcox, MD, MSc, ${ }^{1}$ \\ David Shultz, MD, PhD, ${ }^{2}$ Richard Farb, MD, FRCPC, ${ }^{3}$ Gelareh Zadeh, MD, PhD, FRCSC, and \\ Mark Bernstein, MD, MHSc, FRCS(C) ${ }^{1}$
}

Divisions of ${ }^{1}$ Neurosurgery and ${ }^{3}$ Neuroradiology, Toronto Western Hospital; and ${ }^{2}$ Department of Radiation Oncology, Princess Margaret Cancer Centre, Toronto, Ontario, Canada

OBJECTIVE Multiple meningiomas account for $1 \%-10 \%$ of meningiomas. This study describes epidemiological aspects of the disease and its management, which is more challenging than for single tumors.

METHODS A consecutive series of adult patients with $\geq 2$ spatially separated meningiomas was reviewed. Patients with neurofibromatosis Type 2 were excluded. The authors collected clinical, imaging, histological, and treatment data to obtain information on epidemiology, management options, and outcomes of active treatment and surveillance.

RESULTS A total of 133 consecutive patients were included over 25 years, with a total of 395 synchronous and 53 metachronous meningiomas, and a median of 2 tumors per patient. One hundred six patients had sporadic disease, 26 had radiation-induced disease, and 1 had familial meningiomatosis. At presentation, half of the patients were asymptomatic. In terms of their maximum cross-sectional diameter, the tumors were small $(\leq 2 \mathrm{~cm})$ in $67 \%$ and large $(>4 \mathrm{~cm})$ in $11 \%$ of the meningiomas. Fifty-four patients had upfront treatment, and 31 had delayed treatment after an observation period (mean 4 years). One in 4 patients had $\geq 2$ meningiomas treated. Overall, $64 \%$ of patients had treatment for 142 tumors -67 with surgery and 18 with radiotherapy alone. The mean follow-up was 7 years, with $13 \%$ of treated patients receiving salvage therapy. Approximately 1 in 4 patients who underwent surgery had $\geq 1$ WHO Grade II or III meningioma. Meningiomas of different histological subtypes and grades in the same patient were not uncommon.

CONCLUSIONS Multiple meningiomas are often asymptomatic, probably because the majority are small and a significant proportion are induced by radiation. Approximately two-thirds of patients with multiple meningiomas require therapy, but only one-third of all meningiomas need active treatment. The authors recommend surveillance for stable and asymptomatic meningiomas and therapy for those that are symptomatic or growing.

https://thejns.org/doi/abs/10.3171/2017.2.JNS162608

KEY WORDS multiple meningiomas; radiation-induced meningioma; asymptomatic meningioma; craniotomy; surveillance; oncology

$\mathrm{M}$ ENINGIOMAS are common brain tumors, accounting for approximately one-third of all primary brain tumors in adults. The majority are single. Multiple meningiomas are defined as the presence of $\geq 2$ spatially separated synchronous or metachronous lesions, and they occur in up to $10 \%$ of meningiomas. ${ }^{15,18}$ They can be sporadic, radiation induced, or familial. The 2 familial syndromes commonly associated with the disorder are neurofibromatosis Type 2 (NF2) and familial meningiomatosis.

The pathogenesis, natural history, and management of single meningiomas have been extensively studied; however, similar knowledge on multiple meningiomas remains limited. These lesions represent a heterogeneous group with different etiologies, probably constituting distinct entities from single meningiomas. In addition, multiple meningiomas pose special management challenges because the clinician has to decide whether the disease is symptomatic, which lesion is responsible, if treatment is warranted, which treatment option is best, when to initiate treatment, and how to manage incidental multiple meningiomas.

The literature on this subject is relatively scarce. Most published papers are case reports or small case series, and there is a gap in the knowledge on the best management of this disease. The objective of this study was to review our practice to answer the aforementioned questions and to provide guidance for the management of patients with multiple meningiomas.

\section{Methods}

This is a retrospective observational study of a consecutive cohort of patients with multiple meningiomas 
managed at Toronto Western Hospital between 1991 and 2016. The study received approval from the local Research Ethics Board. Our Neurosurgery Division has a dedicated neuro-oncology service and a multidisciplinary team of clinicians with expertise in the management of meningiomas that serves a population area of approximately 5 million.

Inclusion criteria were adult patients ( $\geq 18$ years) with diagnosis of $\geq 2$ separate meningiomas at presentation or diagnosis of a single meningioma at presentation who developed additional meningiomas during follow-up; and multiple meningiomas of sporadic, radiation-induced, and familial (non-NF2) types. Exclusion criteria were patients with NF2 types (multiple meningiomas and bilateral or unilateral vestibular schwannoma), because their management is more complex and usually focuses on the schwannomas; patients without follow-up data; or those who developed metachronous meningiomas deemed to be metastases or recurrence of a previous meningioma. The latter cases included patients with high-grade meningiomas who developed meningiomas at follow-up or patients who developed tumors in the vicinity of a previously resected meningioma, irrespective of grade.

The meningiomas were deemed radiation induced when the Cahan criteria were fulfilled as follows: the patient had received radiation therapy; the neoplasm occurred in the previously irradiated field; a latency period of several years had elapsed (5 years in the original description); the tumor was histologically and/or radiographically different from the primary; and syndromes associated with neoplasms had been excluded. ${ }^{9}$

The Radiology Department database was searched using the words "multiple" or "second" or "additional" or "another" and "meningioma" or "meningiomas" for the study period, and all of the search results $(\mathrm{n}=2735)$ were reviewed. The following retrospective data were collected for the list of eligible patients: demographic data (age and sex), etiology, presentation (symptomatic or asymptomatic), imaging findings, histological characteristics, and treatment method and outcome. We categorized the tumors based on size, according to their maximum crosssectional diameter (small $[\leq 2 \mathrm{~cm}]$, medium $[>2$ and $\leq 4$ $\mathrm{cm}]$, and large [> $4 \mathrm{~cm}]$ ), and location (convexity, midline [parasagittal, falcine/tentorial, intraventricular], or skull base). Management options included upfront treatment or a wait-and-see approach. Treatment included resection or radiation therapy, in the form of stereotactic radiosurgery (SRS) or fractionated radiotherapy. Adjuvant radiotherapy was administered in selected cases. The outcome for treated patients was local control measured by the need for further intervention for the recurrent tumors. The outcome for patients managed expectantly was tumor growth and development of symptoms measured by the need for treatment during the surveillance period.

The statistical analysis was performed with XLSTAT version 2016.04.32523 (copyright Addinsoft 1995-2016). The descriptive statistics of the clinical, imaging, and histological data were calculated. Continuous parametric variables were analyzed with the unpaired t-test, and categorical variables were analyzed with the Fisher exact test. The significance level was set at $\mathrm{p}<0.05$.

\section{Results}

During a 25 -year period, there were 147 potentially eligible patients. Patients were excluded for missing data (n $=8)$, discharge at presentation $(n=5)$, and mortality in the postoperative period ( $\mathrm{n}=1$, pulmonary embolism). We included 133 patients in the final analysis, with a total of 448 meningiomas. The baseline characteristics of the cohort are summarized in Table 1 . There were 29 men and 104 women with a mean age at presentation of 58 years (range 18-91 years). One hundred six patients had sporadic disease, 26 patients had radiation-induced meningiomas, and only 1 case was associated with familial meningiomatosis. All patients with radiation-induced meningiomas underwent cranial radiation therapy many years before presentation, most in childhood for a different brain tumor. The vast majority of meningiomas were synchronous tumors $(\mathrm{n}=395,88 \%)$, and 34 patients developed 53 metachronous meningiomas.

Patients with multiple meningiomas in our series presented at similar ages compared with those with single meningiomas, but the female/male ratio was higher (3.5:1) compared with the $2: 1$ ratio reported for single lesions $(\mathrm{p}=0.002){ }^{40}$ The female/male ratio was 4.6:1 for sporadic and 1.9:1 for radiation-induced multiple meningioma cases $(p=0.11)$.

\section{Presentation and Imaging}

The modes of presentation were as follows. Sixty-six cases were discovered incidentally and were asymptomat-

TABLE 1. Epidemiological characteristics of 133 patients with 448 multiple meningiomas

\begin{tabular}{|c|c|}
\hline Characteristic & No. $(\%)$ \\
\hline \multicolumn{2}{|l|}{ Sex } \\
\hline Male & $29(22)$ \\
\hline Female & $104(78)$ \\
\hline Mean age, yrs & 58 \\
\hline \multicolumn{2}{|l|}{ Etiology, patients/tumors } \\
\hline Sporadic & $106(80) / 333(74)$ \\
\hline Radiation induced & $26(20) / 110(25)$ \\
\hline Familial meningiomatosis & $1(1) / 5(1)$ \\
\hline \multicolumn{2}{|l|}{ No. of meningiomas } \\
\hline Mean per patient & 3 \\
\hline Synchronous & 395 \\
\hline Metachronous & 53 \\
\hline \multicolumn{2}{|c|}{ Size at presentation, synchronous } \\
\hline Small & $264(67)$ \\
\hline Medium & $87(22)$ \\
\hline Large & $44(11)$ \\
\hline \multicolumn{2}{|c|}{ Location at presentation, synchronous } \\
\hline Convexity & $156(39)$ \\
\hline Midline & $138(35)$ \\
\hline Skull base & $101(26)$ \\
\hline \multicolumn{2}{|c|}{ WHO grade, operated meningiomas } \\
\hline 1 & $68(78)$ \\
\hline II & $16(18)$ \\
\hline III & $3(4)$ \\
\hline
\end{tabular}


ic, and 67 cases had signs or symptoms attributed to one of their tumors. Seven symptomatic patients (10\%) presented with new-onset seizures. At presentation, patients with radiation-induced and sporadic multiple meningiomas each had a median of 2 and a mean of 3 tumors ( 3.2 vs $2.9 ; \mathrm{p}=$ 0.32 ). A similar proportion of patients in the 2 groups were asymptomatic $(62 \%$ vs $47 \%$; $\mathrm{p}=0.19)$. Metachronous meningiomas were more common in the radiation-induced than in the sporadic group (62\% vs $16 \%$; $<<0.001)$. At the final follow-up, patients with radiation-induced meningiomas were found to have significantly more tumors than those with sporadic multiple meningiomas (4.2 vs 3.1; $\mathrm{p}$ $=0.007$ ).

The number of meningiomas followed a negative exponential curve (Fig. 1); 60 patients had 2 meningiomas, 57 patients had 3-5 meningiomas, and 16 patients had > 5 tumors. Only 7 patients (5.3\%) presented with a single tumor that subsequently developed additional meningiomas during follow-up. Of the 395 meningiomas found at presentation, $264(67 \%)$ were small, $87(22 \%)$ were medium, and 44 (11\%) were large. A total of 156 tumors were located in the convexity, 138 on the midline, and 101 on the skull base.

At presentation, $90 \%$ of patients with large meningiomas were symptomatic. The respective proportions of symptomatic patients with medium (without large tumors) and only small meningiomas were $43 \%$ and $16 \%$. The rate of symptoms was significantly higher in patients with large meningiomas ( $\mathrm{p}<0.001)$, but was not significantly different in those with and without medium $(p=0.11)$ or small $(p=0.76)$ tumors. With regard to the location of meningiomas, the rate of symptoms did not differ significantly between those patients with and without tumors in the convexity $(p=0.09)$, midline $(p=0.15)$, or skull base $(p=0.23)$ (Fig. 2). However, there was a tendency for patients with midline or skull base tumors to report more symptoms than those without tumors in these locations (relative risk 1.3).

\section{Management of Disease}

Approximately 2 in 5 patients were treated at presentation, either because they had symptoms $(n=50)$ or because they had large tumors that were expected to become symptomatic $(\mathrm{n}=4)$. The remaining 79 patients were followed and $31(39 \%)$ required treatment at a later stage, principally because their tumor became symptomatic or grew at a rate that was expected to cause symptoms during the patient's life expectancy (Table 2). The mean length of time from presentation to treatment for this group was 4.4 years, with a range from 7 months to 12 years. Eighteen of the 54 patients who received treatment at presentation required treatment of a different meningioma an average of 5 years after diagnosis (range 9 months to 18 years). Of the 81 meningiomas that were treated at follow-up, 46 were small and 26 were medium size at presentation, whereas 6 were metachronous. The proportion of patients who had treatment of a different meningioma during surveillance was statistically similar for patients who had treatment at presentation and for those who did not $(p=0.58)$. Seventeen patients had symptomatic meningiomas that were not treated at presentation; 10 had treatment at a later stage,

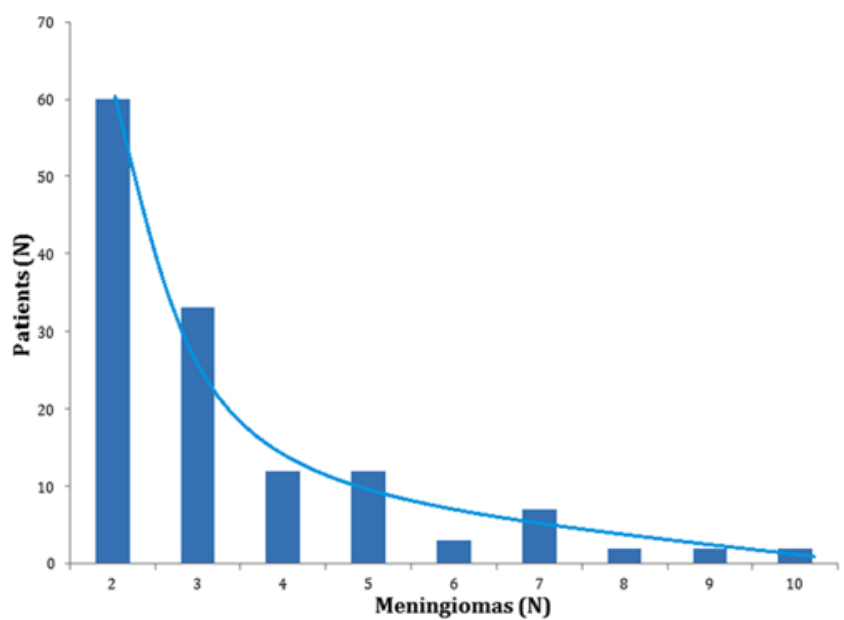

FIG. 1. The frequency of patients with multiple meningiomas decreases as the number of meningiomas increases, with a negative exponential relationship. Figure is available in color online only.

and 7 received symptomatic control measures (e.g., CSF diversion, antiepileptic agents).

Management strategies included surveillance alone for 48 patients, resection of at least 1 lesion for 67 patients, and radiation therapy alone (radiosurgery of fractionated radiotherapy) for 18 patients. Patients with radiation-induced meningiomas were more likely to be treated, either at presentation or during surveillance, compared with those with sporadic tumors (22 of 26 [85\%] and 62 of 106 [58\%], respectively; $p=0.01$ ). Nine patients had $>1$ meningioma removed during the same operation, and 9 patients had radiation therapy for multiple meningiomas in the same session.

Sixty-seven patients had surgery for a total of $87 \mathrm{me}-$ ningiomas, with 51 patients undergoing surgery at presentation and 16 during surveillance. Five patients with highgrade meningiomas received adjuvant radiotherapy. At presentation, 39 of 55 operated meningiomas were large, 13 were medium, and 3 were small. At follow-up, 5 of 32 operated meningiomas were large, 12 were medium, and 10 were small when initially diagnosed; an additional 5 were metachronous. Eleven patients had repeat treatment for recurrent disease, but the majority (84\%) had good control for an average 8.5 years of follow-up (range 0.5-23 years). Among the 67 patients who underwent surgery, 9 underwent radiotherapy (mainly Gamma Knife) for a different meningioma. Finally, among these 67 patients, 150 of 261 meningiomas required no treatment.

Twenty-seven patients underwent radiotherapy for 55 meningiomas as first-line treatment, 10 patients at presentation and the rest during surveillance. Eighteen of these patients had radiation therapy only and the other 9 had both radiation therapy and surgery for different meningiomas. Nineteen patients were treated with Gamma Knife SRS, 6 with fractionated radiation therapy, and 2 with a combination of both. All tumors that received SRS were small or medium and were mostly located at the midline or skull base, with none requiring repeat treatment.

Overall, 85 of 133 (64\%) patients and 142 of $448(32 \%)$ meningiomas were treated, with a mean follow-up of 7 years (range 1-17 years), and 13\% of treated patients un- 


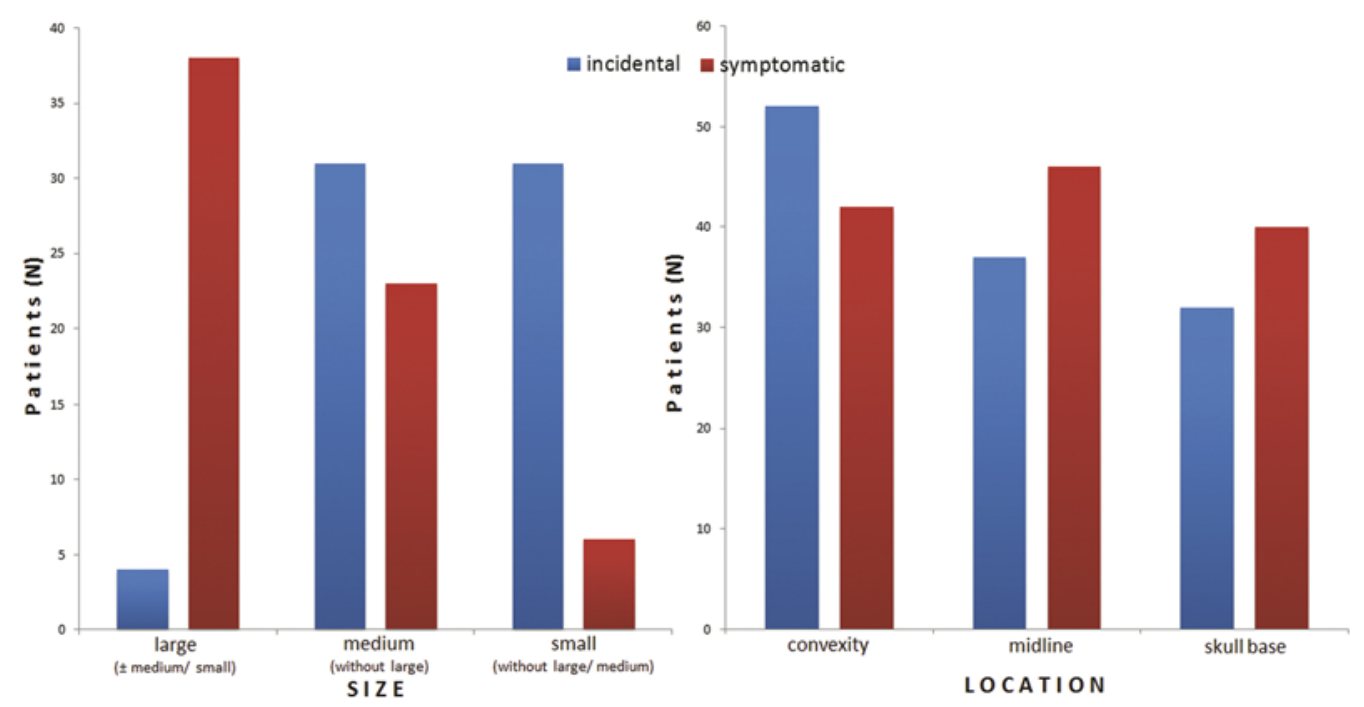

FIG. 2. The distribution of symptomatic and incidental meningiomas in relation to size (left) and location (right). Left: The 3 groups of patients are distinct (patients with large, medium without large, or only small meningiomas). Right: There is overlapping in the groups, because patients with meningiomas in one location may also have tumors in other locations. Figure is available in color online only.

derwent salvage therapy for recurrence. One-quarter of the patients had treatment for $>1$ tumor. Two patients, who had no meningioma treatment, underwent CSF diversion for obstructive hydrocephalus.

\section{Meningioma Histology}

Histological reports were available for all 87 operated meningiomas in 67 patients. The WHO grades were 68 Grade I, 16 Grade II, and 3 Grade III; 73\% of patients had only Grade I tumors, $22 \%$ harbored Grade II tumors, and $5 \%$ had Grade III tumors. The frequency of grades was not significantly different between radiation-induced and sporadic cases $(p=0.64)$. Eighteen patients had resection of $>1$ meningioma (17 patients had 2 tumors resected, and 1 patient had 3 tumors removed); 14 patients had tumors of the same grade (13 Grade I and 1 Grade II meningiomas); 4 patients had tumors of different grades (3 patients had Grades I and II, and 1 patient had Grades I and III meningiomas); 6 patients had meningiomas of different histological type, and 3 had tumors of the same histological type. The histological type was not reported for the remaining 9 patients.

High-grade meningiomas in our series accounted for $23 \%$, whereas the percentage in the literature covering the same time interval varied greatly, from $<5 \%$ to $>30 \%$ in some series. ${ }^{21,31}$ The figure in recent literature was reported as $6 \% .^{12}$ If we assume that the literature refers mostly to single meningiomas, then the proportion of our patients who harbor an atypical or anaplastic meningioma falls within the reported range.

\section{Discussion}

Our results are in accordance with other reports in the literature, which show that the mean age of presentation with multiple meningiomas is in the 6th decade of life and is the same as for patients with single tumors. ${ }^{6,82,28}$
Multiple meningiomas are more common in women, and the female predilection is significantly higher at 3.5:1 than that for single meningiomas. ${ }^{6,8,29}$ In our series, the higher female ratio was also observed in the radiation-induced cases, although the sex difference was less distinct and similar to that for radiation-induced single meningiomas. ${ }^{34}$ It is unclear why female preponderance is significantly higher in multiple lesions, but the recent hypothesis is that hormonal dependency may be higher as suggested by stronger progesterone expression in these tumors compared with their single counterparts. ${ }^{8,35}$ It is possible that genetic factors enhance the potential for tumorigenesis in women, but this potential is somewhat blunted in radiation-induced multiple meningiomas.

Most single meningiomas are sporadic, and the 2 main risk factors of genetic predisposition and prior radiation account for a small fraction of cases. ${ }^{24,40}$ However, this is not the case for multiple meningiomas, and in our series, 1 in 5 patients had radiation-induced tumors. In 2 previous meningioma studies, multiplicity was approximately 7 times more common in radiation-induced compared with sporadic cases. ${ }^{25,26}$ Radiation-induced neoplasia is a stochastic effect of ionizing radiation and the risk of carcinogenesis is higher, especially with therapeutic doses of radiation. ${ }^{36}$ The increased relative risk of multiplicity of

TABLE 2. Management of patients with multiple meningiomas

\begin{tabular}{lcc}
\hline \multicolumn{1}{c}{ Management } & $\begin{array}{c}\text { No. of Patients/No. } \\
\text { of Meningiomas }\end{array}$ & $\begin{array}{c}\text { No. of Patients } \\
\text { w/ Recurrence }\end{array}$ \\
\hline Treatment at presentation & $54 / 61^{*}$ & 10 \\
\hline Treatment during observation & $31 / 59$ & 1 \\
\hline Observation (no treatment) & $48 / 306$ & $\mathrm{NA}$ \\
\hline NA = not applicable. \\
* Eighteen patients from this group had treatment of an additional 22 menin- \\
giomas during surveillance.
\end{tabular}


meningiomas might be explained by the direct biological effects of high-dose irradiation, such as increased genomic instability. In our study, the higher number of meningiomas per patient in the radiation-induced compared with the sporadic group was probably due to the enhanced potential of tumorigenesis caused by radiotherapy.

The 2 familial disorders linked to multiple meningiomas are NF2 and the syndrome of familial multiple meningiomas. The latter is very rare, and we only had 1 case. ${ }^{1} \mathrm{We}$ included it in our study because the management of the disease focuses on the meningiomas rather than the schwannomas, as in NF2. The difference in management compared with other patients with multiple meningiomas is the need for genetic counseling. This is an autosomal dominant trait, and genomic profiling should be considered to assess the risk of tumor occurrence in other family members. ${ }^{30}$

\section{Presentation and Imaging}

Half of our patients were asymptomatic at presentation, in keeping with the fact that most meningiomas are incidental findings and half of all meningiomas are discovered at autopsy. ${ }^{23,32}$ The widespread use of neuroimaging has increased the reported incidence of meningiomas. ${ }^{39}$ The majority of the tumors in our study, as in similar studies, were small and asymptomatic. ${ }^{8}$ The frequency of symptoms was proportional to the size of tumors, with the largest tumors being more symptomatic. This was expected because the mode of presentation of meningiomas is usually by virtue of their mass effect.

The distribution of meningiomas based on location was similar to those reported in other studies. ${ }^{6,8} \mathrm{We}$ found that the rate of symptoms was not significantly different between tumor locations. However, there was a trend toward more symptoms in patients with skull base and midline tumors compared with those with convexity tumors alone. Concordantly, it has been reported that the most common location for meningiomas in asymptomatic patients is the convexity. ${ }^{20}$ In our case, the lack of statistical difference may be a reflection of low statistical power, because a post hoc power calculation estimated that a total sample size of 300-500 patients would be required to detect differences.

Half of our patients had 2 tumors at presentation, with a mean of 3 tumors, as has been found in similar studies. ${ }^{6,8}$ To our knowledge, this study contains the largest sample size, and we demonstrated that the number of meningiomas per patient follows a reverse exponential distribution. This shows that the frequency of patients decreases exponentially with the increasing number of meningiomas; this has also been noted in brain metastases, which are often multiple. $^{5}$

\section{Management of Multiple Meningiomas}

Not all patients with multiple meningiomas require treatment. We applied similar management principles to patients with single meningiomas and treated those with symptomatic meningiomas or growing asymptomatic meningiomas that were likely to cause symptoms. Overall, we treated two-thirds of our patients, and the remainder did not require any active intervention during a mean followup of 7 years. Two-thirds of treated patients had treatment at presentation and one-third after an average observation period of 4 years. In total, only one-third of all meningiomas were treated, because most multiple meningiomas were small and asymptomatic. Our management approach is supported by a similar study by Huang et al., where 59 of 95 meningiomas in 29 patients were removed because of symptoms or growth. ${ }^{8}$ This strategy is also suggested for single meningiomas. ${ }^{43} \mathrm{~A}$ challenge with multiple meningiomas may be identification of the responsible tumor.

Surgical removal was the main form of therapy. In some patients, $>1$ meningioma was resected during the same procedure, mainly because the additional tumors were readily accessible during the approach to the symptomatic one. The radiation-induced meningiomas were more likely to be treated compared with sporadic tumors, because the former generally exhibit more aggressive clinical behavior. ${ }^{37}$ Patients who had treatment at presentation did not have a higher risk of having another meningioma treated during observation compared with those who did not; therefore, more vigorous surveillance for these patients on this basis is not necessary.

Gamma Knife radiosurgery was the treatment of choice for small and some medium-size symptomatic or growing meningiomas with high surgical risk. We also treated spatially separate tumors during the same session. Our longterm results were very good and comparable with those reported in similar studies. ${ }^{28}$ Radiosurgery seems to be an attractive therapeutic option for multiple meningiomas up to $3 \mathrm{~cm}$ in diameter, but more research is needed to establish its effectiveness and safety for this disease.

The rate of repeat therapy in our series, which served as a proxy of clinical recurrence, was $13 \%$. In the study by Huang et al., the rate of radiological recurrence was $29 \%$, which was almost double that of single meningiomas. ${ }^{8}$ Therefore, it may be safe to assume that the rate of radiological recurrence in our study was higher than that of the population of patients with single meningiomas. ${ }^{38}$ This may be due to the bigger proportion of high-grade and radiation-induced meningiomas, or merely the higher tumor burden per patient. Nevertheless, this is one of the reasons that these patients should have close and lengthy follow-up.

We followed most of the asymptomatic patients with serial imaging. This approach identified the minority of patients who warranted therapy and avoided overtreatment. There are studies in the literature on treatment of asymptomatic meningiomas with radiosurgery, even without documented growth. ${ }^{10,27}$ These studies argue that prophylactic radiosurgery is well tolerated and has low morbidity, and risk factors other than tumor growth should be taken into account during decision making. Conversely, in a study specific to multiple meningiomas treated with SRS, the complication rate was $10 \% .^{28}$ In our opinion, patient- and tumor-specific factors should be considered, and there are several advantages to an expectant management approach for radiologically stable incidental meningiomas.

\section{Meningioma Histology}

Our results agree with those of previous studies suggesting that there is no difference in the WHO grades of multiple and single menigniomas. ${ }^{6,8,29} \mathrm{We}$ also found that among patients who had $\geq 2$ meningiomas removed, 
the majority had different histological subtypes, and approximately 1 in 5 even had tumors of different grades. Other authors have also shown that the same patient can have meningiomas of different histology and grade. ${ }^{8,17,19}$ Some claim that this constitutes evidence of different origins of tumors from multiple foci, and that multiplicity is not the result of cell migration through the subarachnoid space. ${ }^{4,17,19}$ On the other hand, several studies support the theory of clonal spread from a single tumor. . $^{8,13,14,22,29,33,42}$ Borovich and Doron suggested that multiple meningiomas have independent origins if their morphology differs, and are of the same origin if it is similar. ${ }^{2}$ In our group, we had cases in both of these categories. However, we appreciate that the origin of multiple meningiomas is probably more intriguing and not explained solely on the basis of microscopic appearances. We believe that both theories may apply in different cases; further research on the genetics of multiple meningiomas will shed more light on the controversy of their pathogenesis.

\section{Limitations of the Study}

The main limitation of our study is its retrospective nature, which accounts for the selection bias in the treatment of patients and a degree of data attrition. In addition, our group was heterogeneous because we included patients with different etiology of disease. To mitigate this, our decision to treat was based on factors other than the pathogenesis of the disease. Another limitation is that the histological grade of the meningiomas in our cohort was based on criteria from different epochs, given that the WHO classification of brain tumors had been revised a few times during the study period, and the relevant rate of all high-grade cranial meningiomas in our hospital was not available for direct comparison. For this reason, our results were compared with those of studies that covered the same time interval. Finally, this is a single-institution case series from an academic hospital in a socialized health care system. However, to our knowledge, this study is the largest of its type, allowing for more powerful statistical analysis and generalizable results, and providing a comprehensive management strategy with details on epidemiology, decision making, and treatment options. ${ }^{8,16,28,41}$

\section{Study Implications and Future Research}

Multiple meningiomas pose special management challenges with regard to diagnostic workup, treatment, and follow-up. Currently, there are a limited number of articles on the natural history and treatment of this disease. We showed that the epidemiology has differences and similarities compared with that for single meningiomas. Our results can inform the decision-making process during consultation with patients. Multiple meningiomas are frequently discovered incidentally. Our philosophy is to treat the symptomatic or the potentially symptomatic tumors to avoid unnecessary procedures and related complications. Our approach is also resource friendly in an era of increasing financial pressures on health care systems.

Currently, there is no effective medical management for meningiomas. There have been suggestions that antiprogesterone therapy may be effective in selected patients with multiple meningiomas due to high expression of progesterone, but this treatment has yet to be tested in prospective clinical trials. ${ }^{35}$ More recently, genetic analysis has identified potential therapeutic targets that can be tested in biomarker-driven clinical trials. ${ }^{3}$ This work involved mainly single meningiomas, but the molecular basis of multiple meningiomas can be fundamentally different; moreover, there are even genetic differences among sporadic, radiation-induced, and familial cases of multiple meningiomas. ${ }^{7,11,33}$ Laboratory research is required to characterize the genetic profile of these tumors, as well as to identify predictors of natural history and response to therapy, and possible actionable molecular targets.

\section{Conclusions}

Multiple meningiomas are more often sporadic, but a significant proportion is radiation induced. The rate of WHO high-grade tumors is similar to single meningiomas. In half of patients with multiple meningiomas, the disease is asymptomatic at presentation. The main form of therapy is surgery, either at presentation or during surveillance, but radiation therapy also plays an important role. However, only a fraction of meningiomas may need treatment, because the majority are small and asymptomatic. We recommend treatment for symptomatic or growing tumors, and observation for asymptomatic and radiologically stable tumors. Further research is needed to elucidate the genomics of multiple meningiomas and to explore the options of systemic therapy.

\section{Acknowledgments}

We thank Alex Kostynskyy, research coordinator, for providing the Radiology Department database of patients with multiple meningiomas and James Hodson, statistician from University Hospitals Birmingham NHS Foundation Trust, for his advice on the statistical analysis.

\section{References}

1. Antinheimo J, Sankila R, Carpén O, Pukkala E, Sainio M, Jääskeläinen J: Population-based analysis of sporadic and type 2 neurofibromatosis-associated meningiomas and schwannomas. Neurology 54:71-76, 2000

2. Borovich B, Doron Y: Recurrence of intracranial meningiomas: the role played by regional multicentricity. J Neurosurg 64:58-63, 1986

3. Brastianos PK, Horowitz PM, Santagata S, Jones RT, McKenna A, Getz G, et al: Genomic sequencing of meningiomas identifies oncogenic SMO and AKT1 mutations. Nat Genet 45:285-289, 2013

4. Butti G, Assietti R, Casalone R, Paoletti P: Multiple meningiomas: a clinical, surgical, and cytogenetic analysis. Surg Neurol 31:255-260, 1989

5. Delattre JY, Krol G, Thaler HT, Posner JB: Distribution of brain metastases. Arch Neurol 45:741-744, 1988

6. Domenicucci M, Santoro A, D’Osvaldo DH, Delfini R, Cantore GP, Guidetti B: Multiple intracranial meningiomas. J Neurosurg 70:41-44, 1989

7. Heinrich B, Hartmann C, Stemmer-Rachamimov AO, Louis DN, MacCollin M: Multiple meningiomas: Investigating the molecular basis of sporadic and familial forms. Int J Cancer 103:483-488, 2003

8. Huang H, Buhl R, Hugo HH, Mehdorn HM: Clinical and histological features of multiple meningiomas compared with solitary meningiomas. Neurol Res 27:324-332, 2005

9. Huvos AG, Woodard HQ, Cahan WG, Higinbotham NL, 
Stewart FW, Butler A, et al: Postradiation osteogenic sarcoma of bone and soft tissues. A clinicopathologic study of 66 patients. Cancer 55:1244-1255, 1985

10. Iwai Y, Yamanaka K, Morikawa T, Ishiguro T, Honda Y, Matsusaka Y, et al: [The treatment for asymptomatic meningiomas in the era of radiosurgery.] No Shinkei Geka 31:891-897, 2003 (Jpn)

11. Joachim T, Ram Z, Rappaport ZH, Simon M, Schramm J, Wiestler OD, et al: Comparative analysis of the NF2, TP53, PTEN, KRAS, NRAS and HRAS genes in sporadic and radiation-induced human meningiomas. Int J Cancer 94:218-221, 2001

12. Kshettry VR, Ostrom QT, Kruchko C, Al-Mefty O, Barnett GH, Barnholtz-Sloan JS: Descriptive epidemiology of World Health Organization grades II and III intracranial meningiomas in the United States. Neuro Oncol 17:1166-1173, 2015

13. Larson JJ, Tew JM Jr, Simon M, Menon AG: Evidence for clonal spread in the development of multiple meningiomas. J Neurosurg 83:705-709, 1995

14. Lomas J, Bello MJ, Alonso ME, Gonzalez-Gomez P, Arjona D, Kusak ME, et al: Loss of chromosome 22 and absence of NF2 gene mutation in a case of multiple meningiomas. Hum Pathol 33:375-378, 2002

15. Lusins JO, Nakagawa H: Multiple meningiomas evaluated by computed tomography. Neurosurgery 9:137-141, 1981

16. Memon MY: Multiple and familial meningiomas without evidence of neurofibromatosis. Neurosurgery 7:262-264, 1980

17. Mocker K, Holland H, Ahnert P, Schober R, Bauer M, Kirsten H, et al: Multiple meningioma with different grades of malignancy: case report with genetic analysis applying single-nucleotide polymorphism array and classical cytogenetics. Pathol Res Pract 207:67-72, 2011

18. Nahser HC, Grote W, Löhr E, Gerhard L: Multiple meningiomas. Clinical and computer tomographic observations. Neuroradiology 21:259-263, 1981

19. Neuss M, Westphal M, Hänsel M, Herrmann HD: Clinical and laboratory findings in patients with multiple meningiomas. Br J Neurosurg 2:249-256, 1988

20. Olivero WC, Lister JR, Elwood PW: The natural history and growth rate of asymptomatic meningiomas: a review of 60 patients. J Neurosurg 83:222-224, 1995

21. Pearson BE, Markert JM, Fisher WS, Guthrie BL, Fiveash JB, Palmer CA, et al: Hitting a moving target: evolution of a treatment paradigm for atypical meningiomas amid changing diagnostic criteria. Neurosurg Focus 24(5):E3, 2008

22. Petrella R, Levine S, Wilmot PL, Ashar KD, Casamassima AC, Shapiro LR: Multiple meningiomas in a patient with constitutional ring chromosome 22. Am J Med Genet 47:184-186, 1993

23. Radhakrishnan K, Mokri B, Parisi JE, O’Fallon WM, Sunku $\mathrm{J}$, Kurland LT: The trends in incidence of primary brain tumors in the population of Rochester, Minnesota. Ann Neurol 37:67-73, 1995

24. Rogers L, Shrieve D, Perry A: Intracranial meningioma: fractionated radiation therapy perspective, in Chin LS, Regine WF (eds): Principles and Practice of Stereotactic Radiosurgery. New York: Springer, 2008, pp 257-270

25. Rubinstein AB, Shalit MN, Cohen ML, Zandbank U, Reichenthal E: Radiation-induced cerebral meningioma: a recognizable entity. J Neurosurg 61:966-971, 1984

26. Sadetzki S, Flint-Richter P, Ben-Tal T, Nass D: Radiationinduced meningioma: a descriptive study of 253 cases. J Neurosurg 97:1078-1082, 2002

27. Salvetti DJ, Nagaraja TG, Levy C, Xu Z, Sheehan J: Gamma Knife surgery for the treatment of patients with asymptomatic meningiomas. J Neurosurg 119:487-493, 2013

28. Samblas J, Luis Lopez Guerra J, Bustos J, Angel GutierrezDiaz J, Wolski M, Peraza C, et al: Stereotactic radiosurgery in patients with multiple intracranial meningiomas. J BUON 19:250-255, 2014
29. Sheehy JP, Crockard HA: Multiple meningiomas: a long-term review. J Neurosurg 59:1-5, 1983

30. Shen Y, Nunes F, Stemmer-Rachamimov A, James M, Mohapatra G, Plotkin S, et al: Genomic profiling distinguishes familial multiple and sporadic multiple meningiomas. BMC Med Genomics 2:42, 2009

31. Simon M, Boström J, Koch P, Schramm J: Interinstitutional variance of postoperative radiotherapy and follow up for meningiomas in Germany: impact of changes of the WHO classification. J Neurol Neurosurg Psychiatry 77:767-773, 2006

32. Staneczek W, Jänisch W: Epidemiologic data on meningiomas in East Germany 1961-1986: incidence, localization, age and sex distribution. Clin Neuropathol 11:135-141, 1992

33. Stangl AP, Wellenreuther R, Lenartz D, Kraus JA, Menon AG, Schramm J, et al: Clonality of multiple meningiomas. J Neurosurg 86:853-858, 1997

34. Strojan P, Popović M, Jereb B: Secondary intracranial meningiomas after high-dose cranial irradiation: report of five cases and review of the literature. Int J Radiat Oncol Biol Phys 48:65-73, 2000

35. Touat M, Lombardi G, Farina P, Kalamarides M, Sanson M: Successful treatment of multiple intracranial meningiomas with the antiprogesterone receptor agent mifepristone (RU486). Acta Neurochir (Wien) 156:1831-1835, 2014

36. Tubiana M, Feinendegen LE, Yang C, Kaminski JM: The linear no-threshold relationship is inconsistent with radiation biologic and experimental data. Radiology 251:13-22, 2009

37. Umansky F, Shoshan Y, Rosenthal G, Fraifeld S, Spektor S: Radiation-induced meningioma. Neurosurg Focus 24(5):E7, 2008

38. van Alkemade H, de Leau M, Dieleman EMT, Kardaun JWPF, van Os R, Vandertop WP, et al: Impaired survival and long-term neurological problems in benign meningioma. Neuro Oncol 14:658-666, 2012

39. Vernooij MW, Ikram MA, Tanghe HL, Vincent AJPE, Hofman A, Krestin GP, et al: Incidental findings on brain MRI in the general population. N Engl J Med 357:1821-1828, 2007

40. Wiemels J, Wrensch M, Claus EB: Epidemiology and etiology of meningioma. J Neurooncol 99:307-314, 2010

41. Wong RH, Wong AK, Vick N, Farhat HI: Natural history of multiple meningiomas. Surg Neurol Int 4:71, 2013

42. Zhu JJ, Maruyama T, Jacoby LB, Herman JG, Gusella JF, Black PM, et al: Clonal analysis of a case of multiple meningiomas using multiple molecular genetic approaches: pathology case report. Neurosurgery 45:409-416, 1999

43. Zygourakis C, Garcia R, McDermott M: Meningiomas, in Bernstein M, Burger M (eds): Neuro-Oncology: The Essentials, ed 3. New York: Thieme, 2014, pp 375-390

\section{Disclosures}

The authors report no conflict of interest concerning the materials or methods used in this study or the findings specified in this paper.

\section{Author Contributions}

Conception and design: Tsermoulas. Acquisition of data: Tsermoulas, Wilcox. Analysis and interpretation of data: Tsermoulas, Wilcox. Drafting the article: Tsermoulas, Turel, Zadeh. Critically revising the article: all authors. Reviewed submitted version of manuscript: Tsermoulas, Turel, Wilcox, Shultz, Farb, Bernstein. Approved the final version of the manuscript on behalf of all authors: Tsermoulas. Statistical analysis: Tsermoulas.

\section{Correspondence}

Georgios Tsermoulas, Queen Elizabeth Hospital, Mindelsohn Way, Edgbaston, Birmingham B15 2GW, United Kingdom. email: georgios.tsermoulas@nhs.net. 